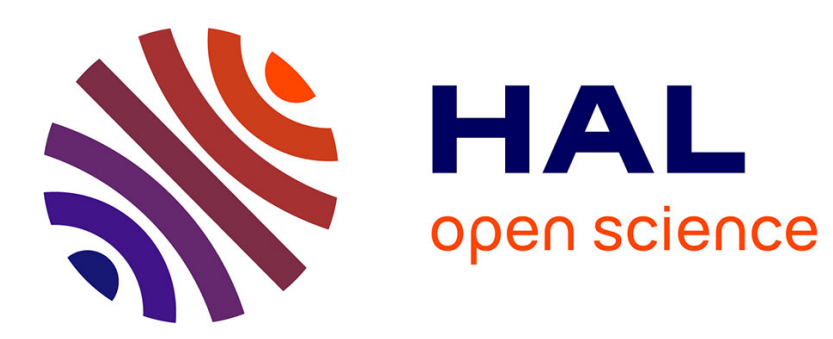

\title{
Des procédés néologiques euphémiques et quelques domaines privilégiés d'apparition
}

\author{
Jean-François Sablayrolles
}

\section{To cite this version:}

Jean-François Sablayrolles. Des procédés néologiques euphémiques et quelques domaines privilégiés d'apparition. La linguistique: revue internationale de linguistique générale, 2016, Néologismes empiriques, 52-2, pp.187-200. 10.3917/ling.522.0187 . hal-03085944

\section{HAL Id: hal-03085944 \\ https://hal.science/hal-03085944}

Submitted on 22 Dec 2020

HAL is a multi-disciplinary open access archive for the deposit and dissemination of scientific research documents, whether they are published or not. The documents may come from teaching and research institutions in France or abroad, or from public or private research centers.
L'archive ouverte pluridisciplinaire HAL, est destinée au dépôt et à la diffusion de documents scientifiques de niveau recherche, publiés ou non, émanant des établissements d'enseignement et de recherche français ou étrangers, des laboratoires publics ou privés. 
HAL JFS 61 2016f/

Sablayrolles Jean-François (USPC et laboratoire HTL UMR 7597)

«Des procédés néologiques euphémiques et quelques domaines privilégiés d'apparition » actes du colloque, Néologismes euphémiques, Saint Jacques de Compostelle, 16 octobre 2015 Montserrat Lopez Diaz et Nuria Rodriguez Pedreira org.,

La linguistique $\mathrm{n}^{\circ}$ 52-2, décembre 2016, Montserrat Lopez Diaz et Jean-François Sablayrolles éd., p. 187-200

Résumé

Paradoxalement, les néologismes dont la nouveauté attire souvent l'attention sur eux peuvent avoir comme fonction d'euphémiser ce qu'ils dénomment. Sans que les procédés de création lexicale et les domaines des référents dénommés coïncident exactement, on constate des liens privilégiés entre les deux. Les périphrases lénifiantes abondent dans le politiquement correct pour dénommer des handicaps divers (déficient visuel...) ou des statuts ou professions dévalorisés (technicien de surface...) ; des innovations d'emploi ont une valeur apotropaïque pour évoquer des réalités déplaisantes ou effrayantes (les banlieues...); des emprunts disent l'origine étrangère et la mauvaise influence extérieure subie dans l'importation d'actes criminels (skimming, smash and grab...) ou de conduites déviantes ou à risque (balconing...). Mais l'usure guette ces néologismes euphémiques comme le montre le sens actuel de décéder signifiant étymologiquement «partir » et employé pour éviter mourir, dont il est devenu un synonyme.

Mots-clés : néologie, euphémisme, emprunt, périphrase

\section{Des procédés néologiques euphémiques et quelques domaines privilégiés d'apparition}

\section{Introduction}

Beaucoup de néologismes ont une portée polémique et agressive (ils abondent par exemple dans le domaine politique qui n'est pas un monde de bisounours ${ }^{1}$ : ce n'est pas gentil de traiter un adversaire de serial menteur ou un ancien ami de capitaine de pédalo ${ }^{2}$, etc.). Mais, quand on regarde de plus près, on observe aussi des néologismes euphémiques, qui peuvent passer inaperçus, puisque c'est précisément leur fonction que de dissimuler des réalités qu'on ne veut pas nommer directement pour de multiples raisons. Certains procédés néologiques, comme l'emprunt, la périphrase, des changements d'emploi... semblent particulièrement utilisés dans cette fonction que l'on voit à l'œuvre dans certains domaines précis. Des liens privilégiés entre des types de procédés et des domaines peuvent même être établis, quoique les deux ne se recouvrent pas exactement. Le politiquement correct conduit à renommer, surtout par des périphrases certains handicaps, activités professionnelles ou statuts sociaux. On tente aussi de contourner des craintes que provoquent certaines réalités par des dénominations neutres, souvent elliptiques ou faisant appel à des hyperonymes ou mots généraux. Un certain nombre de forfaits, de conduites déviantes ou à risques sont nommés, aujourd'hui comme hier, par des emprunts, immotivés dans la langue cible et affirmant l'importation étrangère de ce dont il est question, qui ne pourrait pas être autochtone. La

\footnotetext{
${ }^{1}$ Voir Sablayrolles, 2016.

${ }^{2}$ Le premier, dû à Jean-Marie Le Pen, leader d'extrême droite, visait Jacques Chirac, candidat à sa réélection à la présidence de la République en 2002 et le second a été émis par Jean-Luc Mélenchon (ancien membre du parti socialiste) à propos de François Hollande lors de la campagne présidentielle de 2012.
} 
réception de ces néologismes euphémiques et leur circulation méritent aussi quelques réflexions.

\section{Des périphrases lénifiantes et politiquement correctes}

Le politiquement correct qui s'applique aussi bien à des caractéristiques physiques, qu'à des activités professionnelles dévalorisées ou à des statuts sociaux ou juridiques susceptibles d'être réprouvés recourt d'une manière privilégiée à des périphrases lénifiantes et euphémiques.

\subsection{Handicaps ou caractéristiques physiques (exemples déjà un peu anciens)}

Nombre de handicaps ont été rebaptisés et on remarque la circulation moindre d'un certain nombre de mots qui les dénommaient. On ne parle plus guère en effet de gueule cassée, de cul-de-jatte, d'unijambiste, ou même d'aveugle, de sourd, etc. Cela fait des années du moins que je n'ai pas entendu ou lu les premiers de ces mots. Il est vrai qu'avec la disparition, du fait de leur âge nécessairement avancé, des blessés des deux guerres mondiales, le terme gueule cassée n'a plus beaucoup de raison d'être, mais il n'y a pas de noms régulièrement utilisés pour les personnes blessées à la face et défigurées, par suite d'accidents ou de maladies, que l'on opère parfois maintenant avec des greffes de visage ${ }^{3}$.

Sont englobés dans les personnes à mobilité réduite les handicapés moteur, ainsi que les personnes amputées d'une ou des deux jambes. Et le mot estropié a également disparu de la circulation ${ }^{4}$. Il a été remplacé par un emprunt venu du monde du sport : handicapé (v. $3^{\text {ème }}$ partie). Pour ce qui est des unijambistes et culs-de-jatte, on ne nomme plus directement la perte de membre, mais on remonte à la cause (« il a été amputé d'une ou des deux jambe(s)») ou on prend en compte son effet («il a des difficultés de locomotion »). Dans ce dernier cas, on occulte la perte d'un ou des deux membres inférieurs puisque les difficultés de locomotion peuvent être dues à bien d'autres raisons (perte d'équilibre, faiblesse des articulations, etc.). Alors que les anciennes dénominations 'essentialisaient' la déficience physique : la personne qui en était atteinte se réduisait à cela, la dénomination personne à mobilité réduite qui connaît un emploi exponentiel considère d'abord la personne comme personne. Sa déficience physique et les difficultés qui en découlent arrivent en second.

Quant aux sourds et aux aveugles, on parle souvent actuellement de déficient auditif ou mal entendant, et de déficient visuel, mal voyant, et aussi, plus rarement de non voyant. Il est vrai que ces dénominations permettent d'établir une échelle dans le handicap. Il n'en demeure pas moins qu'à une extrémité de cette échelle, la surdité totale et la cécité existent et qu'il y a des sourds et des aveugles, même si on ne les nomme plus toujours aussi directement. Ces composés ou préfixés concurrents des mots hérités sont de création récente si l'on se fonde sur la date de la ou des premières attestations dans les archives de presse (il arrive qu'une attestation unique précède de plusieurs années la seconde). Mal entendant et

\footnotetext{
${ }^{3}$ Un rapide sondage dans les archives de la presse française disponibles sur le site Europresse confirme en grande partie ces intuitions, mais une étude plus fine de l'évolution de la circulation de ces termes au cours du temps serait nécessaire. Si la surreprésentation de défiguré (10 425 articles en tout dont 786 la dernière année) face à gueule cassée (1 068 et 157) ne signifie pas grand chose du fait de la polysémie de défiguré, celle de amputé des deux jambes (1 457 et 83 ) face à cul-de-jatte (712 et 20) est, elle, très significative. Il faudrait aussi faire une enquête sur la connaissance par les jeunes générations de gueule cassée et cul-de-jatte. Beaucoup de jeunes doivent les ignorer. Les résultats pour unijambiste et amputé d'une jambe sont moins probants (2 482 face 1883 et 170 face à 107), mais sans doute y a t-il des utilisations d'amputé sans précision ou d'hyperonymes (handicapé...) qui montreraient une perte de vitesse pour unijambiste.

${ }^{4}$ Les archives de presse sont éloquentes : 975 résultats en tout, dont 64 la dernière année pour estropié face à 2386 et 159 handicapé moteur et surtout 4500 et 526 personne à mobilité réduite.
} 
mal voyant sont attestés la première fois en 1988 et ont leur attestation suivante en 1992. Déficient visuel apparaît en 1996, avec des emplois rares les premières années. La première attestation de déficient auditif remonte à 2011 et la seconde à 2012. Un écart de 4 ans sépare la première occurrence de non entendant de la suivante : 1994 et 1998.

Mais, par une sorte d'hyper politiquement correct, des locuteurs, de peur d'employer les mots qu'ils ont en tête et sur le bout de la langue et qui sont réprouvés comme stigmatisants en arrivent à créer des monstres linguistiques réjouissants du type déficient audio-visuel! C'était heureusement en l'absence des personnes concernées. Cette hypercorrection en dit long sur le poids de l'interdit social qui règne maintenant, alors qu'il s'agit d'intégrer des élèves ou étudiants souffrant de certains handicaps avec tous les autres.

À côté des mutilations et des déficiences de certains organes des sens, d'autres caractéristiques physiques ne sont plus non plus dites si directement qu'elles l'étaient il n'y a pas si longtemps. Les nains sont ainsi devenus des personnes de petite taille, et les nègres des personnes de couleur. Black sera traité en $3^{\text {ème }}$ partie.

Parallèlement à ces périphrases nouvelles lexicalisées, qui relèvent ou ont relevé — car beaucoup sont déjà installées depuis plusieurs décennies- de la néologie, il existe des périphrases euphémiques qui ne sont pas (encore) lexicalisées et qui ne sont donc pas à proprement parler des néologismes. C'est toute la différence entre la dénomination et la désignation. Si certaines pourront se lexicaliser avec le temps, d'autres sont des hapax conversationnels qui n'ont pas, a priori, vocation à perdurer. Un proviseur parlait ainsi en conseil de classe d'élèves à compréhension lente là où lui-même et des collègues, s'entretenant en privé, disaient idiots, cancres, ou de manière plus imagée et plus récente crêpes ou huîtres... La périphrase permettait de dire en public (représentants d'élèves et de parents d'élèves), de manière acceptable, des réalités qui ne pouvaient pas être dites aussi directement sans risque d'être taxé d'élitisme ou de mépris. D'une manière plus régulière et d'ores et déjà lexicalisée, à défaut d'être encore lexicographiée, on parle d'élèves / d'étudiants en difficulté. Notons encore qu'à propos de ce même type d'apprenant mal comprenant est ludique, ironique et parodique, sur le mode mal voyant, mal entendant... et n'est pas lexicalisé. Il y a ainsi, en discours, de faux euphémismes qui, comme des litotes, donnent plus à entendre que ce qui est dit au pied de la lettre.

Notons néanmoins que des périphrases d'atténuation pour des maladies ont tendance à disparaître au profit des mots justes. Mais alors des mutations sociétales sont en jeu avec un rejet de conduites quelque peu hypocrites dans certains contournements évitant des noms de maladies. Le mot cancer était ainsi en grande partie tabou, et quand il était prononcé, on baissait la voix. On parlait de tumeur maligne pour la maladie et dans les faire-part de décès de longue et cruelle maladie. On affronte maintenant la maladie, sans craindre de dire qu'on en est atteint. Si le spécialiste de cette maladie est, depuis 1920, le cancérologue, il est concurrencé par deux dénominations plus opaques : oncologue depuis 1970 et carcinologue qui n'existe dans le Petit Robert que sous l'entrée cancérologue ${ }^{5}$.

\subsection{Activités professionnelles}

On a beaucoup ironisé, il y a déjà un quart de siècle ${ }^{6}$, sur le remplacement des dénominations anciennes femme / homme de ménage, balayeur par technicien de surface.

\footnotetext{
${ }^{5}$ Le premier de ces noms est encore le plus employé (7 407 articles dont 798 depuis un an) devant oncologue : 2599 et 397 et surtout carcinologue qui n'est présent que dans 3 articles, un de 2002 et deux autres en 2005.

${ }^{6}$ La première attestation de technicien de surface dans la presse figure dans une chronique ironique de Claude Sarraute dans Le Monde en 1989. Mais ses prédictions sur l'échec programmé de ce néologisme ont été démenties par les faits : 635 articles en tout dans les archives de presse (dont 44 la dernière année), sans toutefois
} 
Attribuer le qualificatif de technicien redore peut-être le blason des personnes concernées mais ne change guère en profondeur leur statut social et leur salaire. Il est vrai que c'est sans doute l'époque où se sont développées les entreprises de nettoyage qui emploient des dizaines voire des centaines d'individus, avec du matériel spécialisé que n'utilisent pas la femme de ménage employée dans une maison bourgeoise ou le balayeur des rues qu'est un employé de la voirie. Ce changement de dénomination en dit peut-être plus sur l'organisation du travail que sur les personnes qui le font effectivement. En tout état de cause, il n'est guère employé, dans les conversations courantes, que de manière ironique.

En revanche, toujours dans le domaine du travail, la périphrase euphémique plan social (sous entendu d'accompagnement) qui correspond tout bonnement à un "licenciement collectif », s'est bien implantée au point de figurer dans des articles de dictionnaire à l'entrée social, du fait, malheureusement, des difficultés rencontrées par nombre d'entreprises qui ont recouru à ce qu'on appelait aussi, de manière peu euphémique pour le coup, des dégraissages $^{7}$. Le seul intérêt du changement de dénomination réside dans la recherche, par les partenaires sociaux, de compensations accordées aux salariés qui font les frais de ces restructurations ou cessations d'activités industrielles.

Notons encore que l'instauration, officielle ou non, de ces nouvelles dénominations périphrastiques ne relève pas toujours de l'euphémisme. D'autres raisons peuvent présider à cela, comme la volonté de marquer tout simplement le changement. Ce fut le cas pour un changement de mode de gouvernance, quand les lois de décentralisation de Gaston Deferre ont institué, au début des années 80, les commissaires de la République en remplacement des préfets. Mais on est vite revenu aux préfets, et la dénomination commissaire de la République n'a jamais vraiment circulé dans la langue courante, en dehors des textes administratifs officiels.

\subsection{Statut social}

Outre leurs activités professionnelles, ce sont parfois des statuts sociaux d'individus qui font l'objet de renomination dont la fonction et la raison d'être sont en partie, mais en partie seulement, liées à l'euphémisme. Le cas le plus patent est la substitution de mère célibataire à fille mère. L'opprobre qui visait les femmes que l'on dénommait ainsi disparaît avec une dénomination plus neutre qui met en tête leur statut de mère et en expansion caractérisante le fait qu'elles ne soient pas mariées, alors que la dénomination antérieure mettait en relief le fait qu'elles n'étaient pas mariées et que, malgré cela, elles étaient mères, ce qui était stigmatisé. Les naissances hors mariage étaient en effet condamnées, et le terme fille se chargeait alors de connotations négatives, comme lorsque fille est synonyme de fille de mauvaise vie. Le changement de dénomination s'est accompagné d'un changement de perspective: les mutations sociétales avec la diminution du nombre des mariages, la multiplication des divorces et des familles recomposées ont banalisé les naissances hors mariage (qui sont même devenues majoritaires en France : 57,4\% en 2014) et ont fait voler en éclats les conceptions morales rigides qui dominaient avant mai 68. Si le référent demeure le même, la perception qu'on en a est entièrement différente. Aussi, n'est-il pas complètement opportun de parler d'euphémisme, si ce n'est qu'on peut s'interroger sur la chronologie relative des changements : le changement de dénomination semble s'être développé avant les mutations des comportements et des conceptions. Ceux-ci ont d'ailleurs abouti plus récemment à une nouvelle renomination avec foyer monoparental (ou famille

supplanter homme de ménage avec 1621 articles. Mais il y a en regard 31832 articles contenant femme de ménage.

${ }^{7}$ Ce terme a d'ailleurs provoqué un tollé dans le monde enseignant quand un ministre de l'Éducation nationale Claude Allègre- a déclaré vouloir « dégraisser le mammouth ». 
monoparentale). Ce qui est alors pris en compte n'est plus la naissance dans ou hors mariage, mais le fait qu'une personne est seule pour élever son ou ses enfants, qu'il s'agisse de la mère -c'est le cas le plus fréquent-, mais cela peut aussi être à l'occasion le père.

Dans le domaine du droit, les termes inculpé et inculpation ont semblé indiquer prématurément la faute et la culpabilité (culpa, présent aussi dans battre sa coulpe) alors qu'avant toute condamnation on est présumé innocent. Ils ont été officiellement changés, en 1993, avec une formulation neutre qui adoucit le statut des individus concernés : ils sont mis en examen lors d'une procédure de mise en examen, et ce sont ces termes qui sont employés, même quand les crimes et délits sont avérés et que les personnes ont été prises en flagrant délit ou qu'elles avouent spontanément leur acte, voire le revendiquent ${ }^{8}$.

De la même manière on lit souvent dans la rubrique des faits divers des journaux la mention connu des services de police à propos de victimes d'agressions présentées alors comme des règlements de compte entre membres de bandes rivales. Cette connaissance qu'ont les services de police n'est pas due aux vertus et hautes qualités morales des personnes en cause, mais au contraire à leurs méfaits et délits : ce sont des délinquants. Mais, pour se prémunir de toute suspicion de mise en cause de la présomption d'innocence, les journalistes et leur rédaction n'emploient pas le terme propre délinquant, trafiquant, dealer, etc. mais le contournent avec une expression devenue quasiment synonyme, connu des services de police. Si c'est probablement au départ un groupe syntaxique figurant dans des phrases du type : «la victime est connue des services de police », il semble bien que la récurrence de ce groupe, hors conjugaison verbale et en apposition, a contribué à sa lexicalisation, même si les lexicographes ne l'ont pas encore traité comme tel.

\section{Des innovations d'emploi apotropaïques pour des réalités déplaisantes ou effrayantes}

Une autre raison de recourir à des dénominations euphémiques réside dans le désir d'éviter de nommer trop directement ce qui fait peur de crainte de le faire advenir, de même qu'il ne fallait pas parler du diable, ou qu'on ne nommait certaines réalités que par des antonymes comme la belette la «petite belle » redoutée du fait des ravages qu'elle faisait dans les basses-cours.

\subsection{Des pluralisations non pluralisantes}

Si la mise au pluriel d'un nom est liée à des réalités comptables pour indiquer qu'il y en a plusieurs, il y a au moins deux cas, proches, où la mise au pluriel ne correspond pas à cela : les noms quartier (nombrable) et banlieue (pas toujours non nombrable).

Quand on dit les quartiers, il peut s'agir effectivement de plusieurs quartiers, comme dans « les quartiers récents » opposé aux «quartiers anciens ». Mais, employé sans précision, les quartiers renvoient à un type de quartier : un quartier défavorisé. On peut en effet lire ou entendre «il habite / il vit dans les quartiers ", ce qui n'est pas la même chose que « il habite / vit dans le quartier ». Il s'agit en effet pour les quartiers d'un quartier déshérité, avec des problèmes de chômage et surtout de trafics illicites en tout genre : principalement drogue et armes, avec les rivalités entre bandes pour le contrôle de ce territoire et les violences qui s'ensuivent. On dit aussi pudiquement et euphémiquement quartiers sensibles. Un synonyme quasi parfait est le nom, pluriel lui aussi, les banlieues, alors que pour dénommer les communes entourant une grande ville le singulier est de rigueur : une ville et sa banlieue. La mise au pluriel n'est possible que s’il s'agit de la périphérie de plusieurs villes (« les banlieues des grandes métropoles... »). Et quand on parle des banlieues (d'une ville), il ne s'agit pas de

\footnotetext{
${ }^{8}$ Dans le cas d'euthanasie compassionnelle d'un conjoint ou d'un parent par exemple.
} 
toutes les communes qui environnent telle ou telle ville, mais seulement de celles qui sont défavorisées et où existent des trafics de différentes sortes. Mais cette ellipse d'un caractérisant exprimant trop clairement la situation dramatique de ces lieux a conduit les termes généraux et neutres à se charger de la connotation négative qu'elle était précisément chargée de taire.

\subsection{Autres cas de présentation atténuée de réalités déplaisantes}

Dans ces quartiers et banlieues, mais pas uniquement-là malheureusement, se sont répandues les incivilités (mot encore absent des dictionnaires courants il n'y a pas si longtemps $)^{9}$. Ces conduites inciviles, contraires aux relations de civilité qui devraient régner entre citoyens ne sont pas de simples manquements aux règles de politesse de base (comme ne pas dire bonjour en entrant dans une boutique ou ne pas s'excuser quand on a gêné involontairement quelqu'un, etc.), mais elles renvoient concrètement à des conduites agressives, avec des insultes, des menaces, ainsi que des nuisances avec des comportements qui gênent autrui (tapage diurne et nocturne, consommation de tabac dans des lieux publics, etc.). Le néologisme tend donc à atténuer la gravité des faits en cause, susceptibles de dépôt de plaintes, en les faisant considérer comme de simples manquements aux règles de civilité / politesse ordinaires.

Alors que les personnels chargés de l'hygiène urbaine étaient encore appelés, au milieu du $20^{\text {ème }}$ siècle, dans la langue courante juste familière, boueux, la dénomination éboueur a complètement supplanté cette forme dans l'usage (même si Le Petit Robert ne l'indique pas vieillie). Mais, dans ces deux mots, le radical boue est reconnaissable, avec ses connotations négatives. C'est le terme agent de propreté urbaine qui figure maintenant dans les documents administratifs. Il ne focalise que sur l'aspect positif de la tâche, la propreté, en oblitérant son aspect moins reluisant qui consiste à travailler à débarrasser les ordures, donc à être à leur contact. Régionalement on parlait / parle encore d'ailleurs d'ordurier. Dans le nouveau terme officiel, agent est neutre voire valorisant et propreté inspire des idées ou images de plaisir, de bien-être comme celles que véhiculent les publicités pour les produits d'entretien ménager à la télévision.

\section{Des emprunts pour des délits ou conduites déviantes}

Une autre manière de ne pas dire directement les choses consiste à recourir à des emprunts. D'une part, ces mots ne sont pas (semi)motivés dans la langue cible s'ils le sont souvent dans la langue source. D'autre part, c'est indiquer que les réalités négatives ainsi dénommées ne sont pas autochtones mais viennent de l'extérieur avec de mauvaises influences qui importent des réalités qui ne peuvent pas venir de chez soi. Ainsi la (bonne) qualité des locuteurs de la langue cible reste intacte.

L'absence de motivation de mots dérivés ou composés importés vaut bien sûr pour les gens ignorant la langue source ou ne la maîtrisant qu'imparfaitement. Elle peut être moindre ou effacée pour ceux qui la connaissent bien, mais cela reste néanmoins démotivé dans le système de la langue cible. Par ailleurs, des acronymes et les mots-valises peuvent poser des problèmes encore plus ardus de motivation, comme nous allons le voir.

Ce double éloignement par rapport à ce que serait une dénomination (semi)motivée en langue autochtone est d'un usage ancien comme le montre toute la série des méfaits et crimes portant des noms anglo-américains : gangster, hold-up, kidnapper / kidnapping, pickpocket (voir Jacquet-Pfau et Sablayrolles à paraître, et Napieralski et Sablayrolles 2016). Ont été

\footnotetext{
${ }^{9}$ D'après Wikipedia les premières études françaises sur le phénomène (et donc le nom) datent de 1980.
} 
introduits plus récemment une sorte d'amalgame, le phishing ${ }^{10}$ avec deux équivalents, hameçonnage, ou moins pertinent filoutage qui ne dit pas la spécificité de cette pratique, le pharming ( «logiciel malveillant avec copie de site officiel ayant pour fin de tromper et escroquer »), le spoofing ( «usurpation d'adresse IP »), le skimming («fraude à la carte bancaire en copiant les informations personnelles lors d'un retrait sur un DAB), le smash and grab (avec de multiples traductions sans qu'un élément de ce foisonnement néologique s'impose : défonce et ramasse, fracasser et saisir, tu fracasses et tu saisis, etc.).

Dans les méfaits qui ne recourent pas non plus aux nouvelles technologies, on relève car jacking, go fast, home jacking. Le happy slapping révèle un écart entre un sens compositionnel qui pourrait être positif (happy, mais slap « la gifle » l'est moins, quoique la scène d'un film italien des années 60 où les 'héros' giflent à la volée les voyageurs penchés à la fenêtre du train au moment de son départ soit assez comique) et les réalités dénommées ainsi aujourd'hui : des scènes d'agressions souvent très violentes filmées et diffusées sur internet. L'emprunt swatting (ou allogénisme swating) est plus obscur si on ne sait pas que SWAT est le sigle de Special Weapons and Tactics, l'équivalent du GIGN ou de la BAC en France : il s'agit de fausses alertes présentant des personnalités connues en grand danger (prises en otages par exemple) et faisant se déplacer des troupes d'élite.

Nombre de ces méfaits ont trait à la sexualité. L'euphémisme le plus frappant est sans aucun doute lover boy. Ce 'garçon amoureux' est en fait un souteneur en herbe qui prostitue sa jeune copine auprès de copains de leur âge ou de personnes plus âgées. Il doit s'agir d'un allogénisme puisque, dans ce sens, le terme semble provenir de régions néerlandophones et qu'il est inconnu en anglais. Une formulation connotée plutôt positivement (quoi de plus beau que l'amour !) sert à dénommer, d'une manière opaque, des pratiques délictueuses hautement condamnables. Avec le développement du sexting et de ses conséquences parfois dramatiques avec des suicides sont aussi apparus le revenge porn et le fappening, tous deux ayant comme point commun la diffusion de photos dénudées sans l'autorisation des intéressés et parfois à leur insu. La différence entre les deux tient à ce que le revenge porn concerne des individus quelconques dont un (plus rarement une) ex cherche à se venger en diffusant des photos intimes prises du temps de leur idylle et encore en sa possession, alors que le fappening est la diffusion de photos dénudées de stars à leur insu, par détournement de documents privés. Quant à grooming, c'est employé pour les recherches de jeunes victimes sur internet par des pédophiles.

À côté de ces délits, foisonnent aussi une série de conduites déviantes ou de paris stupides qui se sont développés rapidement via internet et qui portent des noms angloaméricains également. Le binge drinking - où la quantité d'alcool ingéré se transforme en rapidité de son absorption dans les équivalents français : cuite express, biture express, ivresse rapide, etc., apparu vers 2006/2007 en France - connaît un avatar avec la neknomination où on lance sur internet un défi de boire autant d'alcool que soi, filmé sur une vidéo. Un autre défi stupide et dangereux - il a causé plusieurs morts - est le balconing où on se jette du balcon de sa chambre d'hôtel dans la piscine. Il semble qu'il s'agisse d'un allogénisme ou faux anglicisme créé en Espagne, aux Baléares plus précisément. Là encore, des vidéos de ce type d'exploits circulent sur internet, ce qui incite d'autres personnes à faire de même.

Notons encore qu'avec la mode des selfies sont apparus toute une série de termes amalgamés - avec selfie entier ou plus souvent tronqué sous la forme -elfie, -fie voire simplement -ie - de photos de parties de soi qu'on ne met ordinairement pas en valeur (belfie de boot «arrière train » et selfie pour des photos de fesses généralement bien rebondies - la chirurgie esthétique de cette partie du corps est en plein essor -, ou underboobsselfie pour des décolletés inversés, photos de poitrines féminines vues de dessous, pratique qui fleurit en

\footnotetext{
${ }^{10}$ Le mot viendrait de password harvesting fishing.
} 
Thaïlande au grand dam des généraux au pouvoir qui édictent des règlements d'interdiction à ce sujet. Il y a encore des photos de soi dans des situations qui ne sont pas à l'avantage de la personne qui se photographie comme drelfie quand on est ivre (drunk), etc.

Notons encore, pour faire écho à des euphémismes périphrastiques évoqués en début d'article, comme personne de couleur, qu'un autre euphémisme est aussi utilisé avec l'emprunt black qui permet d'éviter nègre et même noir. Le détour par une autre langue fait prendre une certaine distance. Il se passe la même chose avec l'emprunt du monde du sport, hippique au départ, handicapé (hand in cap :'la main dans le chapeau', pour compenser des désavantages) qui s'est substitué, du moins en partie, à estropié, invalide... dont la circulation est devenue très limitée.

La non motivation en langue cible d'un emprunt s'accompagne d'un type particulier de motivation : la forme étrangère dit l'origine étrangère de la chose. Ce n'est sans doute pas par hasard qu'il n'y a guère eu de tentatives de trouver des équivalents français à kidnapping, pickpocket, etc. Le maintien de la forme étrangère est signifiant et tend à dégager la responsabilité de la population autochtone, qui ne fait que subir de mauvaises influences extérieures. Cette atténuation de la responsabilité relève de l'euphémisme.

\section{Conclusion}

Certains de ces euphémismes s'imposent (mise en examen), d'autres suscitent souvent un sourire amusé (technicien de surface pour homme de ménage, balayeur), d'autres encore traduisent de véritables évolutions sociétales tant dans les réalités que les manières de les appréhender (foyer monoparental éclipsant fille-mère tombé en désuétude). Il arrive aussi qu'avec le temps, d'anciens euphémismes perdent cette valeur et deviennent exactement synonymes des lexies qu'ils étaient censés concurrencer : décéder (étymologiquement «partir de quelque part») est depuis longtemps équivalent de mourir, et d'autres formulations apparaissent comme $s$ 'en aller, nous quitter...

Bien d'autres raisons que la dénomination de nouveautés techniques ou scientifiques président à l'apparition de néologismes. Parmi celles-ci, le désir d'un interlocuteur individuel dans une circonstance énonciative donnée ou le besoin d'un groupe social ou de toute la société de nommer différemment des réalités souvent anciennes par courtoisie envers les interlocuteurs ou par des exigences de savoir vivre en société sont à l'origine de créations néologiques euphémiques, pour atténuer ce qu'une formulation directe pourrait avoir de blessant ou de dérangeant.

\section{Bibliographie}

BonhOMME Marc, 2014, Pragmatique des figures du discours, Champion.

JACQUET-PFAU Christine, SABLAYROLLES Jean-François, à paraître, «Tout ce qui vient du monde anglosaxon est-il mauvais ? », Colloque Quo vadis terminologia en hommage à John Humbley, Paris 7, 18-20 février 2015.

NAPIERALSKI Andrzej, SABLAYROLLES Jean-François, 2016, «Emprunts sociétaux : conduites 'délictueuses', excessives ou à risque », Emprunts néologiques et équivalents autochtones Z Hildenbrand, A. Kacprzak et J.-F. Sablayrolles éds., Lambert Lucas, p. 89-157.

SABLAYROLLES Jean-François, 2016, «Les néologismes du domaine politique », La Fabrique des mots français, Lambert Lucas, p. 279-293. 